\section{Survey reveals a positive mindset is key to success}

A whopping $97 \%$ of dental professionals think that a positive mindset is very important when it comes to team success, according to a new survey carried out by NBS Training.

Despite this, the figures collated also revealed that over $50 \%$ have never invested in mindset training, leaving a significant gap in the pursuit of success in dental practice.

Commenting on these results, Tracy Stuart of NBS Training, said, 'With the vast majority of respondents indicating that their mindset has been affected by COVID-19, it is more important than ever before that principals understand changes are needed to their team's mindset. If things stay the same, it will not only affect everyone's mental health but also the practice's bottom line.

'In many cases, the dentist's leadership style needs to change, so that the team can learn to understand how their brain works and, where a new mindset is needed, to make better choices. It's actually very easy to achieve with the right guidance, and, trust me, I understand a thing or two about bringing out the best in people!'

To book a strategy call with Tracy to see how she can help your team members change their mindset for the better, email tracy.stuart@nbstraining. co.uk or call 01438217944.

\section{Easier endodontics for GDPs}

Are you looking for files that put you firmly in control of endodontic treatment? Then look no further!

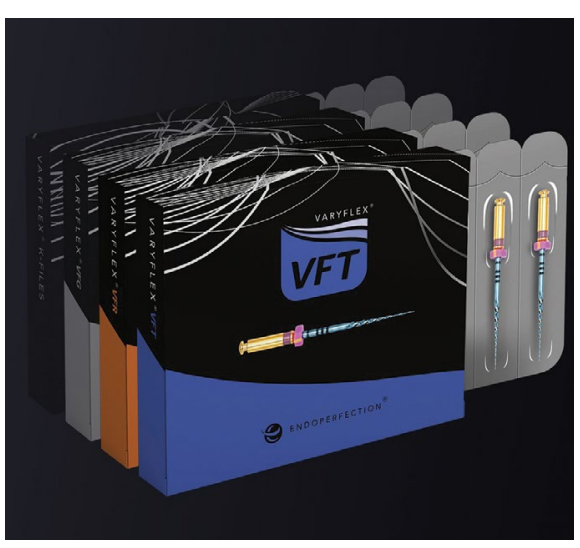

Now available exclusively through Wright Dental, Endoperfection's VaryFlex files offer amazing value without compromising on quality and are simple to use.

For example, the files have incredible flexibility due to the heat-treated nickel titanium (NiTi) alloy and an $11 \mathrm{~mm}$ shank, which is shorter than usual, making working with VaryFlex very comfortable and easy.

Endoperfection is the first UK designed rotary file system and its ethos is to enthuse dentists striving to deliver unsurpassed levels of treatment for their patients.

For further information, visit www.wright-cottrell.co.uk/ new-endoperfection-files-range/.

\title{
Enhancing patient comfort
}

You need a prophy paste you can trust when delivering prophylaxis for your patients.

The Kemdent Prophylaxis Paste features a medium grit and unique formulation that effectively, quickly and easily eliminates food and drink stains from tooth surfaces.

Its viscosity is optimised to improve adherence to the brush for simple handling, while it is thixotropic so as not to spatter for a cleaner and more pleasant patient and practitioner experience. Further improving patient comfort, the product comes in several flavours to meet the preferences of various patients, including original, strawberry, spearmint and bubblegum.

In addition, it is fluoride-free, available in Heavy Duty (coarse) and easily polishable for a smooth finish that both you and the patient will appreciate. To find out more, contact the friendly team at Kemdent today.

For more information about the leading solutions available from Kemdent, visit www.kemdent.co.uk or call 01793770256.

\section{Elevated oral health}

Do you see more female patients then male for preventive-maintenance appointments? Or maybe more men are making enquires into elective treatment to improve their smiles?

This year, it's all about instructing your adult patients in proven oral health practices, based on a solid routine and quality tools. When oral health is good, general heath and wellbeing will be elevated.

TANDEX makes premium products, from daily brushes to interdental brushes and adjuncts. There is also a handy FLEXI educator tool, which you can use to support patients with choosing the right-size brush for each space.

Keep prevention simple and effective recommend TANDEX.

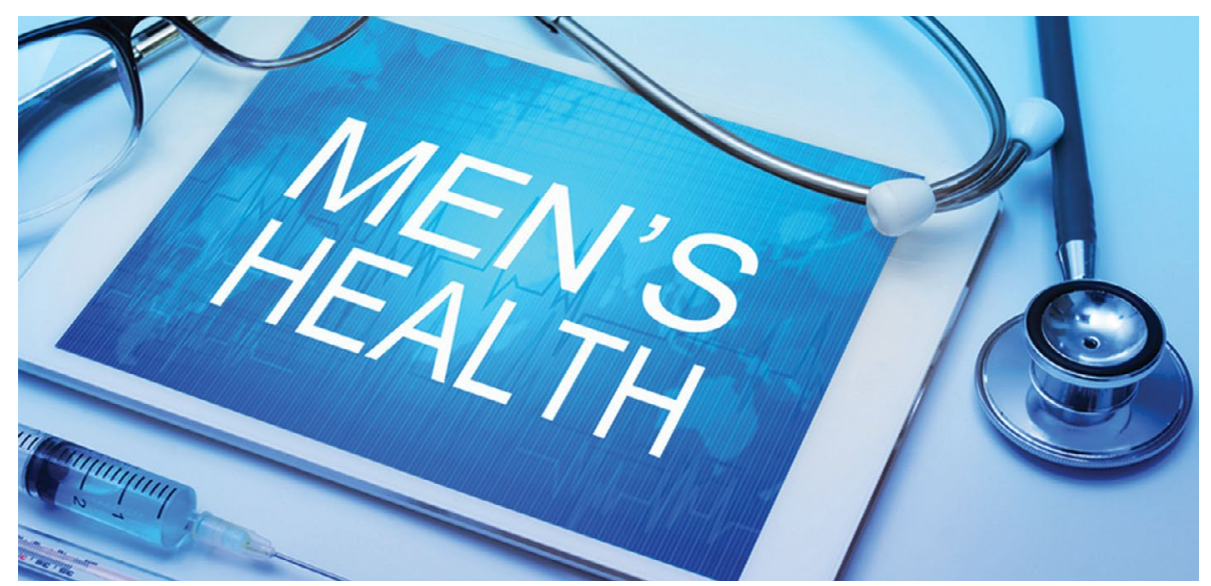

